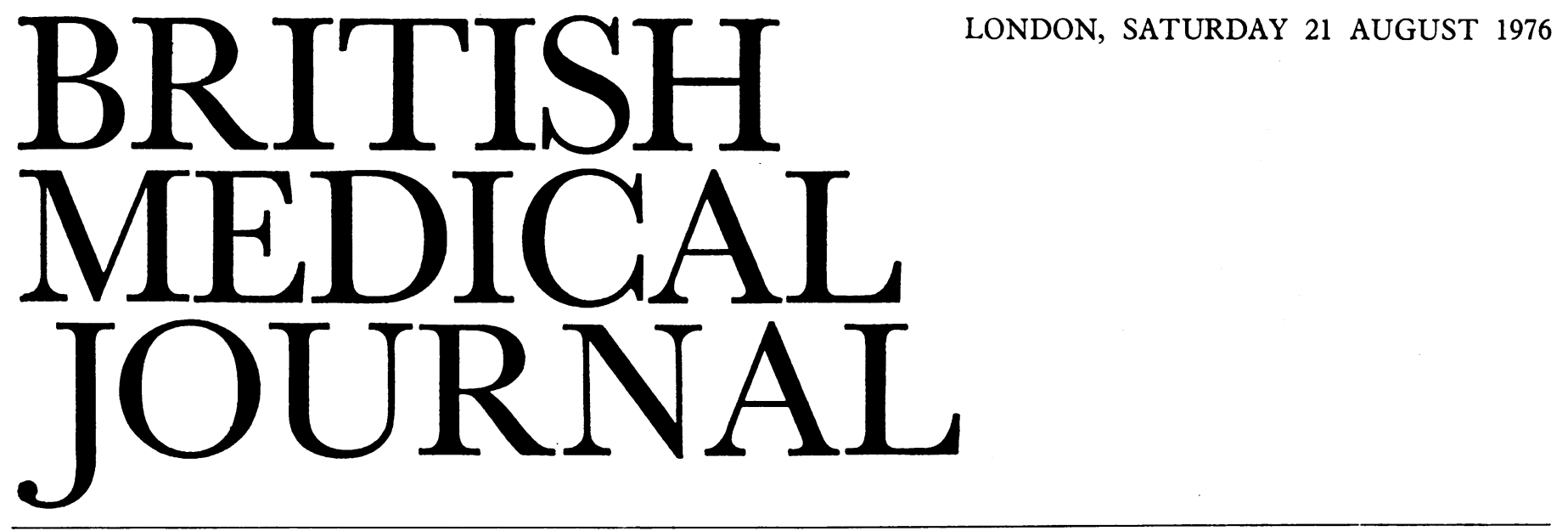

\title{
Lung cancer and smoking: Is there proof?
}

Doctors who advise patients to stop smoking cigarettes are often asked whether it is really proved that smoking is the major cause of lung cancer. Such a question betrays an ignorance of both the existing evidence and what would constitute proof, so that it may be appropriate to review these aspects again. The matter is far from academic, for a causative association indicates a completely safe preventivetreatment for the disease.

There is a striking and undisputed association between cigarette smoking and lung cancer of oat cell or squamous cell type. This is evident whether patients with lung cancer (and controls) are asked about their smoking habits or whether lung cancer mortality is observed in people whose smoking habits have previously been determined. Moreover, the greater the number of cigarettes smoked the higher the risk of lung cancer, and in regular cigarette smokers this is the commonest form of cancer. As in most cancers the incidence of lung cancer in smokers increases rapidly with age, but in ex-smokers it remains remarkably constant-frozen, as it were, around the age the habit was stopped. Together with the increases (over and above those that could be attributed to improved diagnosis) in lung cancer mortality around the world since cigarette smoking became a very common habit, these facts have persuaded most people who have examined the evidence that cigarette smoking causes lung cancer. That is the most plausible interpretation of the epidemiological evidence. Furthermore, cigarette smoke condensate is carcinogenic in laboratory animals.

In allowing that this evidence still does not amount to formal proof of the causative hypothesis, we must point out that what might be regarded as proof is impossible to achieve: a planned experiment in which two randomly selected groups of people were persuaded to take up or to avoid the habit, or alternatively for one randomly selected group of smokers to give up and another to continue to smoke.

What of any alternative views? Burch has received a good deal of attention for resurrecting the view that lung cancer is mainly genetic and that its association with smoking is because both have a similar genetic basis. He must be virtually alone, however, in asserting that the usual hypothesis is "a catastrophic and conspicuous howler"1 which "collapses when all the relevant facts are taken into account." 2 Much stress is placed on the observation that in certain studies the incidence of the disease among heavy smokers is lower in self-described inhalers than in non-inhalers. In fact, only small differences in carbon monoxide uptake after smoking have been found between these groups, with much overlap in levels. ${ }^{3}$ It is possible that the so-called inhaling anomaly reflects differential deposition of the carcinogen between the larger bronchi (where most of the cancers occur) and peripheral parts of the lung. ${ }^{4}$ In this connection it may be relevant that a significantly higher proportion of smokers with peripheral lung cancer claimed that they inhaled than did smokers with central tumours. ${ }^{5}$ It is an interesting exercise to track a course through the evidence in an attempt to sustain the genetic or constitutional hypothesis. Groups such as Mormons, Seventh Day Adventists, and nuns, who do not smoke and who get little lung cancer, must lack the necessary genes. The enormous increase in lung cancer rates this century is inexplicable on this basis and would have to be entirely due to improvements in diagnosis. It then becomes very difficult to explain the persistence of the increasing trend into the period during which diagnostic methods have been reasonably constant. Moreover, to explain on the constitutional hypothesis why the disease should have been so much commoner in men than women (which accords well with the causative hypothesis) would demand not only that doctors had a remarkable prejudice against making the diagnosis in women but that this blindness was sensitive to previous changes in the smoking habits of women. An examination of the sex ratio of the lung cancer mortality rates in different cohorts allows some separation of the effects of smoking from those of improvements in diagnosis. In people born in 1850 who lived before cigarette smoking became widespread this ratio was constant over time, as also it was in people born in 1930-many of whom of both sexes were cigarette smokers. In contrast, people born in 1890 (who included many men but very few women who smoked cigarettes from early adult life) show an increasing trend in this ratio owing to the great excess mortality from lung cancer in men. These analyses provide no support for the constitutional view.

The fact that cigarette smoke contains a carcinogen demonstrable by animal experiments and that in man the organ exposed is the organ affected must be seen merely as a coincidence on the genetic hypothesis. Other observations which cannot be accounted for on this view include the incidence of lung cancer over time in ex-smokers. Since this is unlike that in continuing smokers or non-smokers-or even an imagined mixture of these two groups-it is logical to infer that exsmokers have been affected by their previous smoking and subsequently stopping. ${ }^{6}$ Furthermore, mortality from lung cancer declined in British doctors over the period in which many of them gave up smoking, though we may assume that 
their genes were unchanged. In contrast, in the general population neither mortality rates from this disease nor tobacco consumption fell.

The hypothesis that smoking is the major cause of lung cancer does not imply that genetic ${ }^{7}$ and other factors may not predispose certain smokers to the carcinogenic effects of smoking. But those who publicly assert that people who develop lung cancer were predestined to die from this disease, and that their smoking was irrelevant, do more than ignore the evidence to the contrary: sadly, they may encourage others to smoke cigarettes. Burch has stated that only studies of identical twins with discordant smoking habits will provide a definitive answer to whether the causative or the constitutional hypothesis will explain most cases of the disease. The consequences for being wrong in this matter are serious, and we suspect that doctors will not be attracted by the odds in such a gamble with their patients' lives, even if it is excused by a wish to learn the results of ongoing twin studies which may take decades to complete.

1 Times, 18 August, 1975, p 2.

2 Burch, P R J, Guardian, 22 August, 1975.

3 Wald, N, et al, Thorax, 1975, 30, 113

${ }^{4}$ Doll, R, and Hill, A B, British Medical Fournal, 1964, 1, 1460.

5 Doll, R, and Hill, A B, British Medical fournal, 1952, 2, 1271.

6 Doll, $\mathrm{R}$, fournal of the Royal Statistical Society (Series $A$ ), 1971, 134, 133.

7 Tokuhata, G, and Lilienfeld, A M, Fournal of the National Cancer Institute, $1963,30,289$.

\section{Low spirits after virus infections}

"Tell him to keep his spirits up. Such a nasty depressing complaint. . . ." Thus the rector of Fenchurch St Paul ${ }^{1}$ expressed the common view that influenza and other virus infections predispose to depression, whether immediately or as a late sequel to the illness. As with many firm clinical impressions, proof is remarkably difficult to obtain.

Bacterial infections seem to cause depression in proportion to the severity and duration of the illness in a rational and predictable way. It is also no surprise that virus meningoencephalitis predisposes to psychoses. But the strong impression exists that any virus illness may be accompanied by or be succeeded weeks or months later by incapacitating anxiety or depression, though the illness itself may have been mild and without clinical signs to suggest infection of the central nervous system. ${ }^{2}$ However, this may only show how blunt are the methods for assessing acute disease of the central nervous system.

Influenza, infectious mononucleosis, virus hepatitis, and symptomatic herpes simplex infections have all been accused of causing depression, the evidence being perhaps least convincing for herpes simplex. ${ }^{2}$ An investigation by subjective interview and objective questionnaire of anxiety and depression in unselected patients one year after infectious mononucleosis produced the remarkable finding that women were affected but men were not. This difference in susceptibility to depression and anxiety was evident not only after infection but also before, ${ }^{3}$ so that the infection seemed to unmask or to exacerbate a pre-existing tendency to these disorders. But to generalise from infectious mononucleosis to all virus infections would be unwise, for profound anxiety and depression have been reported after virus hepatitis in patients without any previous tendency to these symptoms. ${ }^{4}$ In this particular study of infectious mononucleosis the identity of the disease was thoroughly proved, but there are grounds for uncertainty about the nature of glandular fever in general, which so easily becomes a convenient label for any febrile illness with lymphadenopathy. Perhaps depression and anxiety call attention to trivial fever and glandular enlargement, or, more probably, a host of unknown viruses may cause glandular-fever-like illnesses with a similar predisposition to psychiatric symptoms.

The good rector's exhortation to "keep his spirits up" often proves lamentably inadequate treatment. The medium to long term prognosis may be good, but at the time depression may be profound and the suicidal risk real. ${ }^{4}$ Explanation and assurance of an eventually complete recovery are important and sometimes sufficient treatment, but a period of treatment with tricyclic antidepressants may be necessary.

1 Sayers, D L, The Nine Taylors. London, Gollancz, 1934.
2 Carranza-Acevedo, J, Pharmakopsychiatrie, 1974, 7, 164.
${ }^{3}$ Cadie, M, Nye, F J, and Storey, P, British fournal of Psychiatry, 1976,
128, 559.
${ }^{4}$ Martini, G A, and Strohmeyer, G, Clinics in Gastroenterology, 1974, 3, 378.

\section{Children with appendicitis}

"When in doubt, take it out" is a well known aphorism relating to the management of patients suspected of having acute appendicitis. As with other useful guidelines, it is best applied by a wise and skilful clinician. Indiscriminate laparotomy every time appendicitis is a possibility would be in no one's best interests. This is particularly so for children, in whom the presentation may be unusual and other conditions readily mimic the symptoms and signs of appendicitis.

Appendicitis is rare below the age of 1 year and thereafter increases in prevalence. Although the incidence is lower in preschool children than in children over the age of 5, the morbidity and mortality are higher. This is partly because of the difficulty of obtaining a clear history and of performing a reliable examination on an ill, frightened young child. There are also differences in presentation. In children the classical early central abdominal colic is not always followed by continuous deep pain in the right iliac fossa. Vomiting is usual but not inevitable. There may be diarrhoea, constipation, or no bowel upset at all.

The difficulties are compounded by the number of other conditions which may mimic appendicitis. Some, such as intussusception, require laparotomy in their own right; others, such as mesenteric adenitis, may require laparotomy to achieve a definite diagnosis. Others should be diagnosed without laparotomy, among them diabetes mellitus, infectious hepatitis, urinary tract infection from the history and examination of urine, and sickle cell disease by the race and the history. But many will be more difficult to diagnose.

In practice gastroenteritis creates much confusion, particularly when the pain precedes either the vomiting or diarrhoea by several hours. It is always worth asking if other members of the family have or have had gastroenteritis recently. Constipation, too, may cause dramatic pain and tenderness in children. A rectal examination, supplemented if necessary by a suppository or disposable enema, should allow the correct diagnosis.

Respiratory tract infections are a frequent cause of confusion. Such infections are themselves common, and while any febrile 